\title{
Arbor
}

\section{Mujeres en Ciencia y Tecnologías Físicas en el CSIC}

\author{
Elvira Moya de Guerra
}

Con la colaboración de Juana Bellanato, Araceli

Flores, María José García-Borge, Beatriz Gato,

Marta I. Hernández, Andrea Jungclaus, Isabel

Márquez, Josefa Masegosa, Ascensión del Olmo

Arbor CLXXII, 679-680 (Julio-Agosto 2002), 549-577 pp.

Presentamos un estudio de la situación de las científicas del área de Ciencia y Tecnologías Físicas en el CSIC. El estudio incluye datos numéricos en cuanto a presencia porcentual de mujeres en las distintas escalas $y$ comisiones de evaluación, asi como testimonios personales, reflexiones y consideraciones generales. Se pone de manifiesto el bajo porcentaje de investigadoras en esta Área. Se concluye el estudio con una serie de recomendaciones.

\section{Introducción y notas generales}

El 22 de noviembre de 2001 empezó nuestra andadura en este proyecto, al que coloquialmente nos referimos como el "Women's Lib de las Físicas del CSIC», en un terreno que era nuevo para nosotras. Además, a lo largo de nuestra carrera científica, la mayoría de nosotras ha trabajado generalmente con hombres, de modo que para algunas este proyecto constituye la única experiencia de un trabajo realizado en colaboración con mujeres. Pues bien, parece que nos ha resultado una tarea altamente gratificante y estimulante y, desde luego, nos ha sido muy fácil ponernos de acuerdo en ella. 
Desde nuestra primera reunión de trabajo quedaron muy claras las prioridades. En primer lugar, este trabajo debería servir para mejorar las condiciones de las científicas y estudiantes que vienen detrás. Por tanto, tendríamos, como principal cometido, hacer propuestas claras y concretas, recomendar vías de solución a los problemas y a las deficiencias con que nos habíamos enfrentado y que observábamos en la actualidad. En segundo lugar, nuestro trabajo debería servir como documento de partida para futuros estudios sobre las científicas en el CSIC. Por tanto, antepondríamos a nuestras ansias literarias y perfeccionistas el cumplimiento del plazo, finalizando el artículo en febrero de 2002. Aceptando de antemano deficiencias, como la fractalidad de los datos y testimonios disponibles, o la formulación imprecisa e incompleta de ideas y conceptos, nos pusimos manos a la obra. Los tres meses que teníamos por delante estaban ya repletos de compromisos en nuestras agendas de trabajo, pero nos comprometimos a sacar tiempo de donde no lo había.

Con esas dos prioridades en mente, estructuramos nuestro proyecto en una primera recogida de datos, experiencias y opiniones que expondríamos con brevedad y claridad, para a partir de ellos, deducir los puntos a destacar y las medidas a recomendar.

En el primer frente -recogida de datos- pretendimos hacer un análisis de la evolución, desde los años 60 ó 70 hasta ahora, de la relación porcentual de mujeres y hombres en los distintos niveles de puestos de investigación en los centros de Física y Tecnologías Físicas del CSIC. Sin embargo, hasta el momento y aunque seguimos trabajando en ello y solicitando ayuda al respecto de la Subdirección General de Recursos Humanos, sólo disponemos de información muy limitada. Presentamos aquí la información global referente al año 2000, que ha sido difundida en el 2001 desde la Presidencia del CSIC y que está disponible en la página web del Consejo, pormenorizada en la distribución por centros. Afortunadamente, una de las aquí firmantes tenía información bastante detallada del año 1994, año en que había realizado un estudio sobre este tema. Pero, ¿y antes del 94? NADA. Bueno, para ser más precisos, casi nada. Afortunadamente, otra vez, una de las colaboradoras había vivido de lleno la situación de la Física en el CSIC entre los años 1970 y 1990 y, aunque no podía darnos números precisos ni estadísticas, sí podía darnos una idea cualitativa de la situación en esa época a través de su propio testimonio y el de algunas antecesoras suyas. Por tanto, en la Sección 2 dedicada a datos recogidos, hemos incluido sólo la èvolución desde 1994. Algo de la situación anterior se vislumbra de los comentarios del apartado de «orígenes» en la Sección 3, pero ésta debería de ser objeto de estudio en el futuro. 


\section{Mujeres en Ciencia y Tecnologías Físicas en el CSIC}

En el segundo frente -opiniones y vivencias- tratamos de obtener el mayor número posible de contribuciones de científicas del área. Obviamente, la información recogida está lejos de ser completa, debido a las limitaciones de tiempo y a la dificultad de comunicación con algunos centros.

Aparte del buen número de contribuciones escritas que se recibieron y que se exponen de forma resumida en la Sección 3, hubo respuestas muy interesantes recogidas en conversaciones. Estas no se hallan reflejadas suficientemente en la Sección 3 y queremos aquí referirnos a ellas porque pueden ser de utilidad para futuros estudios y reflexiones. En concreto, algunas tecnólogas respondieron que no se habían sentido nunca discriminadas en el CSIC, pero sí en trabajos anteriores fuera del Consejo, en particular en empresas de software. Discusión aparte merecen las opiniones de nuestros colegas varones del CSIC y de otros países. Para poner en contexto dichas opiniones, que a continuación comentamos, hay que decir que nos pareció interesante recogerlas aquí debido al carácter predominantemente internacional y masculino del sector de población con el que tenemos que convivir y competir en nuestra actividad científica ordinaria.

En general, nuestros colegas del CSIC se mostraron molestos ante la más mínima alusión a preguntas sobre discriminación por sexo en la Institución, sin dar mayores explicaciones. No obstante, algunos de ellos hicieron hincapié en la idea de que la discriminación en el CSIC no era cuestión de sexo sino de "clase», ya que los órganos dirigentes no daban el debido peso a los científicos y sólo escuchaban a algún «elegido» que variaba según las legislaturas. Así lo hemos oído, así nos parece que debemos transmitirlo por si hubiese lugar a enmendar el presunto fallo.

Ese es pues, el mensaje de nuestros colegas masculinos en el CSIC, y ¿qué pasa con los colegas extranjeros? Destacamos las opiniones mayoritarias de colegas anglosajones, quienes a) no piensan que haya discriminación y, b) señalan que prácticamente sólo en los países mediterráneos hay mujeres científicas en la Física. Claro, hay países anglosajones donde a las niñas se les enseña que no es ésta «una carrera para mujeres», con lo cual no hay discriminación por sexo en la vida profesional porque no hay a quién discriminar. Sin embargo, a menudo se oye comentar a científicas mediterráneas que se sienten más discriminadas por estos colegas. Lo que sí parece claro ${ }^{1}$ es que, comparada con la cultura mediterránea, la anglosajona ha sido más proclive y eficaz en crear un mundo de poder en torno a la investigación científica en el área de Física y en mantener apartadas de ese mundo a las mujeres. Consecuentemente con el lema de que «las cuestiones importantes las resuelven los hombres» y 
atendiendo a un principio de pragmatismo muy anglosajón, su esquema sería: «Si, como Neobía, somos hijas de un Dios Menor, ¿No es acaso más práctico para nosotras y para la especie mantenernos alejadas de conocimientos y esfuerzos que devendrían estériles?»

Obviamente, consideramos que la premisa es falsa y, por ende, la pregunta es falaz. Hoy se acepta universalmente que, a priori, no hay nada en las capacidades intelectuales, creativas y organizativas de una persona que la hagan diferente por el hecho de ser hombre o mujer. Simplemente hay personas que por sus dotes naturales y, sobre todo, por esfuerzos educativos y de su propia voluntad, desarrollan más dichas capacidades. Este desarrollo depende de la medida en que su entorno lo permite. Consideramos que, en lo referente a la cuestión de género, las limitaciones en este sentido vienen más de las condiciones de contorno establecidas por el medio que del potencial individual.

Para terminar con estas consideraciones añadiremos que hay una percepción bastante generalizada de que nuestros colegas masculinos (españoles y extranjeros) tienden a menospreciar nuestras capacidades como científicas. Por tanto, la opinión de los hombres arriba expuesta (que también se contradice con los números de la Sección 2) sería sólo un reflejo de que la percepción de discriminación se da en el sujeto paciente, no en el sujeto agente. No obstante, se percibe, por parte de las más mayores y expertas, una evolución temporal favorable del comportamiento de nuestros colegas en cuestiones de género. Dicha evolución resulta particularmente positiva en aquellos que tienen más vínculos de parentesco y/o de trabajo con científicas. Parece ser que una gran mayoría de físicas del CSIC están casadas con físicos de su mismo instituto o de institutos afines. Este punto puede y debe ser estudiado en el futuro.

Sin duda, hay dos puntos clave que hoy por hoy continúan sin resolverse de forma satisfactoria. Estos problemas transcienden desde luego a la problemática de las físicas del CSIC, aplicándose a toda la sociedad, pero en lo que aquí nos atañe, haremos en la Sección 4 algunas recomendaciones sobre medidas prácticas que el CSIC puede tomar para paliar estos problemas. El primer problema es, sin duda, el de la maternidad. Desdichadamente aún hoy, a principios del siglo XXI, no podemos obviar las observaciones de Planck, cuando a finales del siglo XIX, decía: "I have allowed several women to audit my courses and have had nothing but favourable experience (...), we must keep to the fact that such cases must always be regarded as exceptions (...), it cannot be emphasised enough that nature herself prescribed to the woman her function as mother (...), and that laws of nature cannot be ignored under any circums- 


\section{Mujeres en Ciencia y Tecnologías Físicas en el CSIC}

tances without grave danger - which in the case under discussion would specially manifest itself in the following generation» 2 .

No cabe duda de que hoy por hoy la sociedad tiene recursos muy superiores a los que tenía a finales del XIX para afrontar esta cuestión sin prescindir de la contribución social de la mujer como trabajadora y como madre. Sin embargo, la cuestión está lejos de ser resuelta. Varias de las aquí firmantes que tratamos de desarrollar con éxito no sólo nuestra profesión sino también nuestra faceta de madres, hemos confesado a menudo nuestra pesadumbre ante la carga y la tensión (a veces insoportable) que nos supone la compaginación de estos dos "full time demanding jobs», ser madre y científica. A menudo decimos: «el problema es que cuando estoy a pleno rendimiento, centrada en mi trabajo, tengo la pesadumbre de que estoy abandonando mis deberes de madre de familia y cuando estoy dedicada a mis hijos pienso que abandono mi trabajo». Frases análogas se repiten de generación en generación desde principios del siglo XX, ya que si se consulta cualquier biografía de ilustres antepasadas nuestras, puede verse en todos los idiomas y épocas ${ }^{3}$. Por ejemplo, María Goeppert Mayer (Premio Nobel de Física en 1963 por su descubrimiento del Modelo Nuclear de Capas) decía refiriéndose a sus hijos: «I never stopped feeling guilty, thinking that I should have been home more. Marianne and Peter are wonderful, but I will feel forever that they have missed something... There is an emotional strain due to the conflicting allegiances, that to science and that to the children who, after all, need a mother. I have had this experience in full measure» 4 .

El segundo punto clave radica en la, aún hoy, deficitaria imagen pública de mujer profesional. Percibimos que, hoy por hoy, la imagen de mujer en puestos de responsabilidad provoca un cierto rechazo de forma mayoritaria. Asimismo, percibimos que el éxito profesional de la mujer es peor recibido y aceptado entre su colectivo, que el del hombre. No sólo entre sus pares (que pueden llegar a sentir que el que una mujer ocupe su mismo puesto les desprestigia), sino incluso entre profesionales de niveles inferiores. Aquí el problema está muy relacionado con el hecho de que (desde formas tribales) la idea de respeto y reconocimiento a la superioridad del otro está vinculada al ejercicio autoritario y al sometimiento a la autoridad. Lo cual entra en claro conflicto con el aspecto frágil y formas naturales y amistosas, características de la mujer. Asimismo, la idea de carisma personal se asocia con la de inaccesibilidad e impermeabilidad, en contraposición a los modos de acercamiento personal y confidencialidad más al uso entre mujeres. Aquí la sociedad en general tiene una asignatura pendiente. Para una sociedad en la que tradicionalmente el respeto a la mujer venía dado fundamentalmente en función de su cate- 


\section{Elvira Moya de Guerra}

goría de esposa y madre, es difícil llevar a cabo la incorporación plena del nuevo concepto de mujer profesional. Toda la sociedad está implicada en esta aventura. Las mujeres profesionales tenemos la oportunidad de demostrar nuestras capacidades y talentos, y tenemos el deber de hacerlo con seriedad y responsabilidad. La sociedad at large tiene que comprometerse a aceptar sin cortapisas y a valorar nuestra profesionalidad.

Como se desprende de la lectura de la Sección 3, actualmente, en el sector de mayor nivel educativo en que nos movemos, es difícil observar manifestaciones abiertas de discriminación por sexo; pero sí hay formas sutiles de discriminación en las relaciones profesionales de la mujer científica en el área de Ciencia y Tecnologías Físicas del CSIC, que tienen que ver con los puntos arriba mencionados.

Las sociedades occidentales hemos apostado por la incorporación de la mujer al mundo laboral en todas sus dimensiones. Por tanto, hemos de ser consecuentes buscando vías para resolver temas que están en el fondo minando la aportación, la eficacia y el rendimiento de las mujeres. En una sociedad plural la mujer tiene que aportar su propia identidad y escala de valores, enriqueciendo el mosaico del potencial humano del mundo laboral. Si las condiciones de contorno la obligan o fuerzan a ser «más de lo mismo», limitándose a seguir el patrón masculino previamente creado, entonces el intento no habrá valido la pena y la incorporación de la mujer al trabajo puede tener nefastas consecuencias. Expreso aquí estas ideas aún a sabiendas de que pueden ser objeto de litigio y pueden plantearse de forma muy diferente en otros sectores.

\section{Cuestión de números}

\section{A. Evolución de los porcentajes de mujeres en el Área}

Los resultados que presentamos han sido aportados por los gerentes de los diferentes institutos, por lo que les estamos profundamente agradecidas, y de los que encontramos en la web de los centros. Para poder realizar un estudio comparativo con la evolución durante los últimos años en el CSIC hemos utilizado los datos recopilados por J. Masegosa en 1994. Estos resultados fueron presentados en el curso Mujeres de Ciencia. Presente y Futuro (Universidad de Granada, 1994), gracias a la colaboración desinteresada de la Prof. Carmen Dobarganes, entonces coordinadora institucional en Andalucía. Somos conscientes de que en algunos aspectos no es completo. No obstante, nos da una idea de la realidad actual de nuestro área y de si en los últimos años -donde se supone que vie- 
jos tabúes quedaron anclados en el pasado- hemos obtenido el lugar que como mujeres científicas nos corresponde, o si por el contrario, en algún momento en nuestra historia reciente hemos alcanzado el «techo de cristal»*

En primer lugar, los resultados globales para el área no parecen demasiado optimistas. Tomando los datos aportados por la Presidencia del CSIC y que se pueden encontrar en la web http:/ / www.csic.es, nos enfrentamos a que nuestro área es la más desfavorecida en cuanto a la presencia de mujeres: un 19.9 por ciento, frente a valores típicos en el resto de las áreas por encima del 30\% (exceptuando la presencia de mujeres en el área de Recursos Naturales). Este resultado, analizado aisladamente y sólo para el año 2000, ya puede parecer preocupante, pero más preocupante es el hecho de que el porcentaje es el mismo que el de enero de 1994 (19.2\%). De forma que, si bien creemos que ha habido una dinamización del CSIC en los últimos años, parece que ésta no ha ido en la misma dirección para hombres que para mujeres. Téngase en cuenta que el porcentaje de becarias postdoctorales y predoctorales durante ese mismo año era de un $30 \%$. Nos gustaría aquí resaltar que este estancamiento del CSIC no es exclusivo de nuestro área y que los porcentajes, salvo fluctuaciones de tipo estadístico, son los mismos en todas las áreas a los obtenidos en 1994.

En cuanto a la tendencia general, ya encontrada en 1994, de una diferencia notable a medida que se analiza la presencia de mujeres en escalas más altas, la situación también permanece estable: seguimos con el mismo numero (3) de Profesoras de investigación que en el año 1994, hemos aumentado en 2 el número de Investigadoras científicas (los números obtenidos por nosotras no coinciden con los que hay en la Web, 8 frente a los 5 publicados por la presidencia). De forma que seguimos con nuestro típico 10 por ciento en puestos de mayor responsabilidad y también de poder científico y político, como la presencia en tribunales, jefaturas de departamento y dirección de centros. En cuanto a la escala más baja, a la cual parece que nuestros colegas nos ponen menos reparos, tampoco la situación es muy halagüeña: hemos aumentado en 2 puntos, un 26 por ciento en la actualidad frente al 24 por ciento de 1994 .

Este hecho se reproduce claramente a la hora de ocupar puestos directivos en los diferentes Institutos. En la actualidad no existe ninguna directora en toda el área, y sólo hay una Vicedirectora. Por el contrario, el porcentaje aumenta de manera importante para los Gerentes, de hecho solo 3 Gerentes son hombres, cargos que tradicionalmente han sido

* Barrera invisible que la científica no ve pero con la que se encuentra en su carrera. 
identificados con labores femeninas. El hecho de que exista una fuerte tendencia a que los Directores de centros sean Profesores de Investigación o Investigadores Científicos produce una clara discriminación para las investigadoras del CSIC a la hora de acceder a puestos directivos.

A continuación pasaremos a detallar los resultados obtenidos entre las diferentes disciplinas en los institutos de investigación. En la Tabla 1 se presentan los datos de Institutos propios del CSIC y en la Tabla 2 los de centros mixtos.

En los centros propios del CSIC (Tabla 1) existen dos Institutos singulares con porcentajes muy por encima del valor medio, el Instituto de Óptica y el Instituto de Microelectrónica de Madrid. Sería interesante decir que el aumento de porcentaje femenino en ambos Institutos coincide con una drástica reducción del personal en plantilla a lo largo del periodo 1994-2000. En el Instituto de Óptica, como puede verse en sus anuarios, ha habido siempre una gran participación femenina (por ejemplo, en

TABLA 1. Institutos propios del CSIC: número de mujeres/número total de científicos

\begin{tabular}{|cccccc||}
\hline \multirow{2}{*}{ IN S T $^{*} \cdot$} & C.T. & I.C. & P.I. & TO TA L & $\%$ \\
\cline { 2 - 6 } & $2000(1994)$ & $2000(1994)$ & $2000(1994)$ & $2000(1994)$ & $2000(1994)$ \\
\hline IM A F F & $2 / 9(1 / 4)$ & $1 / 8(0 / 9)$ & $1 / 8(1 / 5)$ & $4 / 25(2 / 18)$ & $16(11.1)$ \\
IO & $2 / 9(2 / 9)$ & $1 / 1(1 / 6)$ & $1 / 1(0 / 2)$ & $4 / 11(3 / 17)$ & $36.4(17.6)$ \\
IEM & $7 / 24(5 / 13)$ & $1 / 8(2 / 8)$ & $1 / 8(1 / 7)$ & $9 / 40(8 / 28)$ & $22.5(28.6)$ \\
IA & $3 / 10(2 / 7)$ & $0 / 4(0 / 3)$ & $0 / 4(0 / 3)$ & $3 / 18(2 / 13)$ & $16.7(15.4)$ \\
IF A & $4 / 15(0 / 3)$ & $1 / 7(0 / 1)$ & $0 / 1(0 / 0)$ & $5 / 23(0 / 4)$ & $21.7(0)$ \\
IA A & $6 / 21(3 / 11)$ & $0 / 7(0 / 4)$ & $0 / 0(0 / 1)$ & $6 / 28(3 / 16)$ & $21.4(18.8)$ \\
IA I & $3 / 14(3 / 13)$ & $1 / 6(1 / 6)$ & $0 / 1(0 / 1)$ & $4 / 21(4 / 20)$ & $19(20)$ \\
C M M N & $5 / 12(5 / 18)$ & $1 / 1(1 / 3)$ & $0 / 2(0 / 2)$ & $6 / 15(6 / 23)$ & $40(26.1)$ \\
T O T A L & $32 / 114(21 / 78)$ & $6 / 42(5 / 40)$ & $3 / 25(2 / 21)$ & $41 / 188(28 / 139)$ & $22.6(20.1)$ \\
\% & $28(27)$ & $14.3(13)$ & $12(9.5)$ & $22.6(20.1)$ & \\
\hline \hline
\end{tabular}

* C.T. (Científico Titular); I.C. (Investigador Científico); P.I. (Profesor de Investigación). Los nombres de los centros se encuentran en el Apéndice. 
el año 1975 la tercera parte de los Profesores de Investigación eran mujeres -6 hombres, 3 mujeres-). Dicho instituto fue reestructurado, disminuyendo notablemente su plantilla en el año 1994 y se nombró a una mujer como directora del centro para proceder a convertirlo en un instituto más competitivo. En cuanto al Instituto de Microelectrónica de Madrid, está formado por dos departamentos: el de Fabricación y Caracterización de Nanoestructuras, con dos mujeres en él de un total de 7 científicos en la plantilla, y el de Fabricación de Dispositivos, Sensores y Biosensores con 4 mujeres de un total de 8.

TABLA 2. Centros mixtos: número de mujeres/número total de científicos, año 2000

\begin{tabular}{|cccccc||}
\hline INST $^{*}$. & C.T. & I.C. & P.I. & TO TA L & $\%$ \\
\hline C A B & & & & $0 / 5$ & 0 \\
IA G & $0 / 1$ & $0 / 1$ & $0 / 0$ & $0 / 2$ & 0 \\
IFIC & $3 / 10$ & $1 / 3$ & $0 / 1$ & $4 / 14$ & 28.6 \\
IFC A & $0 / 3$ & $0 / 2$ & $0 / 0$ & $0 / 5$ & 0 \\
IIIA & $1 / 9$ & $0 / 2$ & $0 / 1$ & $1 / 12$ & 8.3 \\
IN M B & $3 / 12$ & $1 / 6$ & $0 / 1$ & $4 / 19$ & 21.1 \\
IN M S & $2 / 6$ & $0 / 0$ & $0 / 0$ & $2 / 6$ & 33.3 \\
O E & $0 / 1$ & $0 / 0$ & $0 / 0$ & $0 / 1$ & 0 \\
IA C & $0 / 2$ & $0 / 1$ & $0 / 2$ & $0 / 5$ & 0 \\
IE E C & $1 / 1$ & $0 / 3$ & $0 / 1$ & $1 / 5$ & 20 \\
TO T A L & $10 / 45$ & $2 / 18$ & $0 / 6$ & $12 / 69$ & 17.4 \\
\% & 22.2 & 11.1 & 0 & 17.4 & \\
\hline
\end{tabular}

* C.T. (Científico Titular); I.C. (Investigador Científico); P.I. (Profesor de Investigación).

Los nombres de los centros se encuentran en el Apéndice.

En el otro extremo de la balanza se encuentran el Instituto de Matemáticas y Física Fundamental (IMAFF) y el Instituto de Acústica (IA). El IMAFF tiene tres departamentos: Física Atómica y Molecular Teórica (dos mujeres); Partículas, Campos y Cosmología (dos mujeres) y Matemáticas. El IA tiene dos departamentos: Acústica Ambiental (dos mujeres); y Señales, Sistemas y Tecnologías Ultrasónicas (una mujer). 
El resto de los Institutos son bastante homogéneos, con proporciones de mujeres entre un 19 y un 23 por ciento. Sin embargo, es interesante detallar por líneas de investigación en cada uno de los Institutos restantes. El caso del Instituto de Astrofísica de Andalucía (IAA) es particularmente interesante: de las seis investigadoras de este instituto, cuatro pertenecen al Departamento de Astrofísica Extragaláctica y representan el 50 por ciento de su plantilla. Es también resaltable que en dicho departamento el porcentaje de mujeres investigadoras postdoctorales es también el más alto de todo el Instituto, (apuntando en la dirección ya argumentada en el trabajo «Mujeres de Ciencia. Presente y Futuro» de que los centros y departamentos con más mujeres incorporan también más mujeres).

El Instituto de Estructura de la Materia (IEM) es uno de los Institutos más grandes de nuestro área y no se aprecian diferencias obvias en la distribución de los diferentes departamentos. La disminución de un 28.6 a un 22.5 por ciento en los últimos seis años refleja, en parte, la incorporación de un grupo predominantemente masculino procedente del Instituto de Óptica Daza de Valdés (IO).

Por último el Instituto de Física Aplicada es un compendio de investigadores procedentes de otros Institutos, en particular de la reestructuración del IO, lo que queda reflejado en su evolución en los últimos seis años: ha pasado de no tener mujeres a ser éstas el 21.7 por ciento de la plantilla del centro.

En cuanto a los centros mixtos (Tabla 2), los datos sugieren que no son especialmente favorables a promocionar la carrera científica de la mujer. Es resaltable especialmente que estos centros se generan casi siempre a instancias de iniciativas de varones y con escasa participación de mujeres. Sólo cuando dichos centros ya han alcanzado su madurez se empieza a vislumbrar una participación de la mujer similar a la observada en los centros propios del CSIC.

\section{B. Participación de mujeres en tribunales de oposiciones}

Los datos que presentamos en este apartado están elaborados a partir de los tribunales publicados en el Boletín Oficial de Estado, comprendidos entre 1995 y 2001.

En los tribunales para plazas de Científicos Titulares (antes Colaboradores Científicos), la participación media en estos años ha sido de un 16 por ciento. Por años el desglose es el siguiente: 
Mujeres en Ciencia y Tecnologías Físicas en el CSIC

\begin{tabular}{|c|c|}
\hline AÑO & $\%$ \\
\hline 1995 & $16.7 \%$ \\
1996 & $6.7 \%$ \\
1997 & $10 \%$ \\
1998 & $13 \%$ \\
1999 & $18 \%$ \\
2000 & $24 \%$ \\
2001 & $14 \%$ \\
MEDIA & $16 \%$ \\
\hline
\end{tabular}

El porcentaje de mujeres en tribunales de CT en nuestro área debe considerarse bajo. En ningún caso se llega a la media publicada en la Web del CSIC de participación de mujeres en los tribunales, juntando todas las áreas (año 1999 el 34.6\% y año 2000 el 29\%). En el caso de este área de Físicas y Tecnologías Físicas no se cumple ni siquiera la proporción de Científicas Titulares en el área (26\%) y tampoco se ve ningún incremento paulatino en función de los años. Es, por tanto, claro el camino que debería seguir el CSIC en los próximos años: no sólo aumentar la proporción de mujeres en los tribunales en número, sino tener una política más activa que rompa con el estereotipo masculino de la Física.

En los tribunales para plazas de Investigador Científico la media es del 9 por ciento, y aquí el problema es la estadística de números pequeños. Existen años como 1997, 1999 y 2000 en que los tribunales estuvieron compuestos sólo por hombres. El desglose por años se da en la siguiente tabla donde hemos incluido, además del porcentaje, el número de mujeres respecto al total de miembros en los tribunales:

\begin{tabular}{||c|c|c|}
\hline AÑo & $\mathbf{N}^{\circ}$ Muj./N $\mathbf{N}^{\mathbf{T}}$ otal & $\%$ \\
\hline 1995 & $1 / 5$ & $20 \%$ \\
1996 & $1 / 10$ & $10 \%$ \\
1997 & $0 / 7$ & $0 \%$ \\
1998 & $2 / 20$ & $10 \%$ \\
1999 & $0 / 10$ & $0 \%$ \\
2000 & $0 / 10$ & $0 \%$ \\
2001 & $4 / 25$ & $16 \%$ \\
MEDIA & $8 / 87$ & $9 \%$ \\
\hline
\end{tabular}


En los tribunales para plazas de Profesor de Investigación la media es del 9 por ciento. El desglose por años se da en la siguiente tabla:

\begin{tabular}{||c|c|c|}
\hline AÑo & $\mathbf{N}^{\circ} \mathbf{M u j} / \mathbf{N}^{\circ}$ Total & $\%$ \\
\hline 1995 & $1 / 5$ & $20 \%$ \\
1996 & $0 / 7$ & $0 \%$ \\
1997 & $0 / 7$ & $0 \%$ \\
1998 & $1 / 10$ & $10 \%$ \\
1999 & $1 / 10$ & $10 \%$ \\
2000 & $1 / 15$ & $6.7 \%$ \\
2001 & $2 / 15$ & $13 \%$ \\
MEDIA & $6 / 69$ & $9 \%$ \\
\hline
\end{tabular}

Tampoco en el caso de IC y PI se llega, en media, a la proporción de mujeres en el área. Además, se llega a esta proporción por una mayor participación de las mujeres en los tribunales de tecnologías físicas, como es el caso de los tribunales de IC de los años 1998 y 2001.

Creemos que un punto importante de reflexión para las autoridades del CSIC debe ser el hecho de que desde hace diez años tenemos el mismo número (tres) de Profesoras de Investigación en el área.

\section{Cuestión de opiniones: testimonios y percepciones sobre la discriminación por sexo}

\section{A. Orígenes}

En esta Subsección mostramos textualmente, y en primera persona, algunos testimonios comenzando con el de una Profesora de Investigación Emérita.

\section{«MIRANDO HACIA ATRÁS»}

Si empiezo por el principio, he de decir que ya en casa de mis padres no hubo discriminación puesto que éramos tres chicas. Por otra parte, asistí a un instituto de Segunda Enseñanza femenino; por lo tanto, no existía competitividad entre chicos y chicas. En la Universidad ya fue otra cosa. En mi curso, en la Facultad de Química, al empezar en 1944, aproximadamente un tercio éramos muchachas (mi promoción se licenció en 1949). Allí sí recuerdo que experimenté algo de la «dominación mas- 


\section{Mujeres en Ciencia y Tecnologías Físicas en el CSIC}

culina», pero era el ambiente de la sociedad de entonces. Me acuerdo, por ejemplo, de un profesor que prefería chicos para hacer prácticas especiales.

Y terminé la carrera. No sabía qué hacer. Busqué trabajo: en un laboratorio farmacéutico, en donde hice un intento para entrar, sólo querían mujeres como ayudantes, nada de licenciadas. No podían pasar de los estudios de Bachillerato. No lo comprendí. Pensar en la Universidad era una locura. Tenía la sensación de que era inabordable llegar a ser profesora porque en aquella época no sabía de ningún caso. Sí existían de ayudantes de laboratorio, supongo que de forma gratuita. Incluso me ofrecí, pero no tuve éxito.

Para ganar tiempo me matriculé de algunas asignaturas de doctorado y entre ellas, en una de Espectroscopía con el Profesor Miguel A. Catalán. Una de mis hermanas se encontró con el Prof. José Barceló Matutano, con quien yo había estudiado Física y Química en el Bachillerato, que estaba trabajando a tiempo parcial en el casi recién fundado Instituto de Óptica Daza de Valdés (en el Dpto. de Espectroscopía que dirigía el Prof. Catalán) y le dijo que fuese a verle. Yo pensé que de la Óptica sólo me apetecía trabajar en Espectroscopía, por lo tanto no hubo problemas para empezar a hacer la Tesis Doctoral.

Como el Prof. Catalán ya tenía trabajando mujeres en su departamento y el Prof. Barceló acababa de llegar de los EEUU y estaba solo, no tuve problemas de ninguna clase por el hecho de ser mujer. Además, en el mismo grupo entraron pronto otras jóvenes a hacer la Tesis.

Pronto tuve una beca, y después de leer la tesis me nombraron Ayudante de Investigación, luego Colaborador eventual del Patronato «Alfonso X el Sabio», seguido de Colaborador científico del Patronato «Juan de la Cierva»; después, con los años, Investigador y, finalmente, Profesor de Investigación en el Instituto de Óptica.

Puedo decir que en toda mi carrera investigadora no tuve que competir prácticamente con hombres, al menos siendo consciente, aunque sí tuve noticias, a posteriori, de que, en el Instituto de Óptica algunos se enfadaron por adelantarles yo al ser nombrada Investigador y Profesor de Investigación antes que ellos. También, como anécdota, puedo contar que, cuando obtuve una beca para ampliar estudios en Alemania, un compañero le aconsejó al Director que yo no fuese porque «me ahogaba en un vaso de agua» (lo cual no creo que fuese cierto). Me lo confesó él mismo años después. Menos mal que el Director confió en mí.

En resumen, como el Director del Instituto de Óptica, Prof. Otero, no parecía tener nada en contra de las mujeres científicas, como lo demos- 
traba el hecho de que antes que yo habían entrado otras mujeres, entre ellas María Teresa Vigón, María Egüés, y Olga García Riquelme, no puedo decir que encontrase discriminación. Yo por mi parte no tenía ambiciones de "poder", y ahí sí podía haber tenido dificultades.

En realidad, tuve mucha suerte de haber ingresado en el Instituto de Óptica. Sabía que en otros institutos de investigación, dependiendo del director, después de leer la tesis doctoral las mujeres tenían que abandonar el CSIC. En el Instituto de Óptica, cuando esto ocurría, era porque no había puestos de trabajo, lo que, como sabemos, ha sido el problema del CSIC antes y ahora. Yo entré en el momento oportuno.

Respecto a mi estancia en Alemania y después en Inglaterra, las mujeres científicas en los Institutos de Física y Química en que estuve pertenecían al género «avis rara», mucho más escasas que en España. En el Institut für Physikalische Chemie de la Universidad de Friburgo creo que sólo había una mujer haciendo la tesis, en cuyo departamento, por supuesto, me pusieron. Después, ¡no sé por qué razones! trabajó en otro centro. En la hora del café y en las reuniones solamente éramos las dos únicas mujeres. En el Physical Chemical Laboratory de la Universidad de Oxford también había muy pocas mujeres estudiando, yo sólo recuerdo a una polaca. También la hora del café y del té estaban pobladas casi exclusivamente por hombres.

Desde luego, no cabe duda de que en la rama de Ciencias, al menos, la situación era igual o peor en los países europeos que en España. En nuestro país, mucha culpa de la situación la teníamos las mismas mujeres. No teníamos claro si nuestra misión era convertirnos en mujeres científicas. Muchas de mis compañeras de curso, al casarse, se dedicaron a su familia (el mismo marido no dejaba que trabajasen fuera de casa), aunque unos años después algunas buscasen trabajo, principalmente en la Enseñanza, que era más compatible con su vida familiar. También hay que dejar constancia de la dificultad de encontrar trabajo para una mujer casada.

Para terminar, no puedo dejar de mencionar una frase que oí en mis tiempos de estudiante refiriéndose a que nuestra misión era convertirnos en «las mujeres cultas de unos hombres cultos y las madres cultas de unos hijos cultos». Esto estaba en la mente de todos y de todas, y eso ya era una suerte. El destino de otras fue peor porque no les dejaron o no pudieron llegar a la Universidad.

Así pues, mi experiencia como científica del CSIC ha sido de no discriminación. Sin embargo, sé de otras colegas, mayores que yo, que tienen experiencias muy diferentes, como lo demuestra el testimonio de María Egüés, quien nos cuenta a continuación sus experiencias. 


\section{Mujeres en Ciencia y Tecnologías Físicas en el CSIC}

«YO FUI DISCRIMINADA EN EL CSIC POR SER MUJER»

Comencé la carrera de Físicas el curso 1935-1936 en la entonces llamada Universidad Central y terminé el curso a finales de mayo de 1936. Así, la Guerra Civil me encontró con el primer curso de la carrera acabado, pero lo que no sabía era que me esperaban tres años de vacaciones forzosas.

Terminada la contienda, el curso 1939-40 se presentó con la perspectiva de poder hacer dos cursos intensivos para ganar algo del tiempo perdido, en una universidad con los laboratorios arruinados y un cuadro de profesores totalmente diezmado. Por ejemplo, en Físicas solamente quedaba un catedrático titular: D. Julio Palacios. Las asignaturas comunes a Matemáticas estaban mejor dotadas en cuanto a profesores; pero en Físicas el profesorado lo componían, además de D. Julio, auxiliares y ayudantes como encargados de cátedra, cargados de buena voluntad.

Las asignaturas de Electricidad y Física Matemática las impartía el Profesor Durán, que más tarde ganó la cátedra de Óptica y que estaba muy interesado en el porvenir de los físicos españoles. La de Óptica estaba a cargo del Profesor Biel. Con estos profesores terminamos la carrera el curso 1940-41, curso normal sin los agobios de los dos intensivos.

Por marzo o abril de 1940, el Prof. Durán, que al mismo tiempo que sus tareas universitarias trabajaba como ayudante en la sección de Óptica del Instituto de Física y Química de la antigua Fundación Rockefeller - núcleo del proyecto del CSIC entonces en embrión-, me comunicó que en dicha sección se iba a impartir un cursillo de Óptica Geométrica y Cálculo de Sistemas Ópticos a cargo de un profesor alemán (el Prof. Weidert). Su consejo fue que yo tomase parte en él. El jefe de la sección de Óptica del Instituto era D. José María Otero, que también era Subdirector del Laboratorio de Óptica del Ministerio de Marina. Acepté, y, cuando llegó el Prof. Weidert y empezó a impartir su cursillo, fui para asistir al mismo. Allí me encontré con un grupito de ocho o diez personas: licenciados, doctores e ingenieros que iban a lo mismo que yo, y como únicas mujeres, Piedad de la Cierva y yo. Entonces, me dijeron los profesores Otero y Durán que el Director del Instituto, D. José Casares, había permitido que yo asistiese al cursillo, pero en cuanto terminasen las clases debía ausentarme del edificio porque él no quería mujeres en su Instituto. Lo cierto es que había una secretaria y bibliotecaria y Piedad de la Cierva, porque pertenecía al personal del Instituto antes de la guerra. El curso, que se dabä todas la tardes, duraba dos horas, con una pausa de un cuarto de hora. Todo estaba debidamente escrito en unos encerados clarísimos en los c de, aunque no sabíamos alemán, podíamos seguir su exposición. Después, como los que dominaban el alemán eran los pro- 
fesores Otero y Durán, había un repertorio donde nos explicaban todo lo que en el encerado había escrito el Prof. Weidert. Luego había que hacer prácticas de cálculo de sistemas, que entonces se hacía persiguiendo un rayo de luz a través de todas las superficies que constituían el sistema óptico, mediante cálculo logarítmico. Esto se hacía en parejas para evitar en lo posible los errores que pudieran cometerse. Llegamos felizmente al final del cursillo, y entonces me propusieron firmar un contrato con el Ministerio de Marina para, trabajando en el Instituto, dedicarme al cálculo de sistemas ópticos, cosa que acepté de inmediato. Esto requería trabajar mañana y tarde y continuar por las tardes con un nuevo cursillo del Prof. Weidert. Empecé mi trabajo, siempre con el miedo de que me viera D. José Casares en horas no lectivas. Siempre había alguien que avisaba: «iMaruja, que viene D. José!», y yo me escondía donde podía; muchas veces debajo de una mesa de trabajo porque no me daba tiempo de encontrar un sitio mejor. Pero hubo un día en que me topé con él y me echó una bronca de las que no se olvidan. Me dijo, entre otras cosas, que si volvía a verme por allí fuera de las horas del cursillo, me pondría en la calle sin más aviso, que no quería mujeres en el edificio y que a Piedad la toleraba porque hacía tiempo que trabajaba allí y conocía a su familia, mientras que a mí no me conocía de nada. Salí llorando de la entrevista y les dije a los Prof. Otero y Durán que no contasen conmigo porque no pensaba volver más. Entonces buscaron una solución intermedia, que consistía en trabajar por las mañanas en un despachito que tenía el Instituto Torres Quevedo en el Museo de Historia Natural y así poder seguir el cursillo por las tardes. Y eso duró hasta que ocupó la Secretaría General del Consejo D. José María Albareda, en que las cosas cambiaron y las mujeres podíamos entrar libremente en los edificios del Consejo. Y aquí terminó mi odisea, porque después pude simultanear mi puesto en el laboratorio y taller de investigación del E.M. de la Armada con ser Ayudante en la sección de Óptica Geométrica del Instituto Daza de Valdés y, más tarde, Jefe de dicha sección.

"UNAS ANÉCDOTAS ENLAZANDO PASADO Y PRESENTE»

Cuando empecé a hacer el Doctorado en 1970, mi director de tesis decidió que no podía pedir una beca del Ministerio de Educación para mí porque iba a casarme y no llevaría a cabo la realización de mi tesis. Quiero dejar bien claro que lo digo sin acritud, sólo como un testimonio de lo que hace ya más de treinta años era el «status quo». Cuando lo supe, lloré; no por el hecho de que pudiese tener o no la beca sino por lo que me hirió pensar la falta de seriedad que se me suponía, ya que no se me consideraba una persona responsable capaz de cumplir sus compromisos profesionales. 


\section{Mujeres en Ciencia y Tecnologías Físicas en el CSIC}

Recuerdo que entonces, para aplacarme, un querido compañero me dijo que «no lo tomase a mal», que era comprensible que el Profesor no quisiese pedir la beca pensando que al casarme me quedaría embarazada y tendría descendencia, lo que implicaba que, forzosamente, al menos por unos meses, tendría que dejar de trabajar. Yo le contesté que esa posibilidad existía, pero que ese hecho al menos era controlable. Añadí que si esa era la razón entonces no podría pedir la beca para nadie, porque él mismo, por muy varón que fuese, podía tener un accidente y tener que hacer reposo y ausentarse del trabajo durante una temporada. Nunca volvimos a mencionar esa conversación, pero al recordarla ahora me pregunto si, cuando al año siguiente este querido compañero tuvo que ausentarse varios meses del trabajo por haber dado unas vueltas de campana con su coche, el director de tesis o él mismo se impacientaron por el posible incumplimiento del compromiso con su beca.

Como decía, han pasado más de treinta años $\mathrm{y}$, aunque aparentemente han cambiado mucho las cosas, en el fondo no han cambiado tanto.

Debo decir que jamás había vuelto a acordarme de esta anécdota hasta que el otro día, presenciando la oposición de una joven colega, oí a uno de los miembros varones del tribunal preguntar -en un tono que me pareció peyorativo- si era por razones de maternidad el periodo de dos años «en blanco»(que tenía en su currículum) entre los dos sexenios aprobados. Este comentario me pareció igualmente ofensivo que los que yo había oído hace más de treinta años.

¿Me pedís que os cuente más?, pues bien, añadiré que no me arredré. Con una Beca del GIFT (Grupo Interuniversitario de Física Teórica) hice mi tesis doctoral y después mi postdoctorado en el MIT (EEUU). Tuve contratos en el MIT, en el NBI, en la UAM. Entretanto, tuve hijos, y un largo etc. A mi vuelta a España tuve (jclaro está!) que opositar y en el 81 saqué mis oposiciones reglamentarias (¡de las de seis ejercicios!) de Catedrática de Universidad, compitiendo con otros ocho colegas varones «así hemos demostrado que no somos machistas», dijo el, a la sazón, catedrático más antiguo del Área.

Como damnificada de la LRU, y tras sacar una plaza de IC, me incorporé al CSIC, donde hube de vencer una notable resistencia. Pero encontré que el CSIC era "mi casa». Todo por lo que había luchado tanto en la vida era por poder hacer investigación científica con sosiego, tiempo para reflexionar y buen ambiente humano, y aquí, en el Campus de Serrano del CSIC, lo encontré. Formé mi grupo, saqué plaza de PI (1989), gané más internacionalidad, reconocimiento y fama. Mi antiguo director de tesis llegó a decirme que era su discípula más «aventajada» e interna- 
cional. Sé que no es para tanto, que mi carrera está llena de claroscuros, recovecos y obstáculos, y que la carrera científica nunca termina, pero cuando miro atrás me digo aquello de «You've come a long way baby!».

¿Cuál ha sido la clave? Estar segura de dónde quería ir y no dejarme vencer por la adversidad.

¡Ah!, ¿Me preguntáis que si hay discriminación en el CSIC? Pues sí, la hay ¿dónde no? ¿Acaso no somos en cierta medida «machistas» nosotras mismas? ¿Acaso no hay evidencias de diferencias institucionales en el CSIC de trato hombre-mujer a todos los niveles?, ¿Me haríais la pregunta si no las hubiese?¿Alguien diría «así demostramos que no somos machistas» si verdaderamente no lo fuese?

Pero seamos conscientes de que hoy, como hace 20,30, 40, 50 años, seguimos siendo afortunadas de movernos en el sector de población de mayor nivel educativo, lo que hace que la discriminación por sexo sea pecata minuta comparado con lo que pasa en otros sectores. Por eso tenemos también una gran responsabilidad, no sólo la de hacer bien nuestro trabajo, sino, lo que nos es más difícil, la de permear nuestra fuerza y espíritu hacia otros sectores.

\section{B. Resumen de los testimonios personales}

Veamos primero algunos puntos que afloran de las comunicaciones orales y escritas recibidas: la mayoría de las compañeras que han enviado su «experiencia personal» no se ha sentido discriminada en el CSIC, de forma explícita, directa, o denunciable, por el hecho de ser mujer; sin embargo, la mayoría sí ha notado una discriminación indirecta más sutil (consciente o inconsciente), por parte de muchos o algunos de los colegas hombres (y mujeres), así como del personal de apoyo y de talleres, que se pone de manifiesto de maneras muy diversas. Por ejemplo, a través de:

- Formas de infravaloración encubierta: actitudes paternalistas dañinas de algunos jefes o directores de trabajo, dándoles a las mujeres menos responsabilidad y protagonismo intelectual que a los hombres.

- Formas de dirigirse a «ellas» menos respetuosas que para dirigirse a «ellos» (por ejemplo: «Srtas.», para ellas y «Doctores» para ellos, ¡como sucedió en una oposición!).

- Comentarios maliciosos con doble rasero descarado dependiendo de si la víctima es hombre o mujer, normalmente en detrimento de la mujer. En la valoración global de la mujer influye, mucho más 


\section{Mujeres en Ciencia y Tecnologías Físicas en el CSIC}

que en el hombre, sus relaciones privadas (quién es su novio, marido o amante, de qué familia viene...) o sea, se mezcla mucho más su condición profesional con su vida privada. Esto es una consecuencia de que en nuestra sociedad la mujer tiene triple estatus: el que viene de la cuna, el que le confiere su matrimonio y el que ella se gana por méritos propios, siendo éste último, desgraciadamente, el que menos se le valora (excepto en casos excepcionales). El hombre, sin embargo, tiene sólo doble estatus y, aunque la cuna tiene bastante peso, lo que más cuenta y se valora son sus logros personales. Su matrimonio cuenta muy poco o nada en cuanto a su valoración.

- Percepción diferente de un mismo «defecto» dependiendo de si se trata de un hombre o una mujer, de nuevo, normalmente, en menoscabo de la mujer. Un «defecto» que en el caso de un hombre puede no llamar siquiera la atención o no se ve como tal defecto, en una mujer puede resultar fatal y altamente peligroso. El ejemplo clásico sería el del ligón mujeriego, perfectamente aceptable (incluso admirado), mientras que el equivalente femenino, incluso con muchas menos «tablas» que el varón, sería una ambiciosa/arribista/... . Pero hay muchos más ejemplos de los que somos muy poco conscientes. Por ejemplo, a un científico algo «dejado» y muy desordenado se le tiende a ver como muy trabajador, ensimismado en sus ideas, en su laboratorio, en sus teorías...(«un genio»), mientras que a una científica algo «dejada» y desordenada se la tiende a ver como una neurótica, que tiene un lío mental que no se aclara, y que no da abasto con su casa y el trabajo.

Además de lo anterior, la mayoría coincide en la sensación clarísima de que a las mujeres científicas no se nos escucha de la misma manera que a nuestros colegas masculinos, aunque digamos exactamente lo mismo y lo digamos antes. Queramos o no, lo cierto es que, cuando abrimos la boca, nuestras ideas y opiniones cuentan menos que si las expresa un hombre. Dar más crédito a los hombres que a las mujeres, además de injusto, es un síndrome muy generalizado y nos incumbe a todos, ya que también las mujeres tenemos tendencia a padecerlo. Como todo síndrome, normalmente no sucede de forma intencionada (aunque en algunos casos sí lo sea), sino que se origina en algunos mecanismos reflejos de la percepción mental subliminal, lo cual no es de extrañar, ya que todos vivimos sumergidos en una sociedad machista. Según Carmela Valdemoro (que por edad y estatus tiene mucha experiencia), todavía faltan un par de generaciones para que este problema se solucione. El «machismo inconsciente», como algunos lo denominan, tiene una serie de conse- 
cuencias funestas, que van desde lo simplemente desagradable hasta lo traumático. Veamos algunos ejemplos:

- Sentir que «cuenta menos» que sus pares masculinos puede ser devastador para algunas científicas jóvenes que no tienen un puesto estable; y puede provocar fácilmente su retirada profesional. En todo caso, este sentimiento, bastante deprimente y humillante, puede ocasionar problemas psicológicos de cierta consideración, incluso verdaderos traumas, dependiendo de la vulnerabilidad psíquica de la mujer en cuestión, entre ellos, a saber, pérdida de la autoestima, inseguridad para expresarse, tristeza, sentimientos de impotencia y desamparo, agresividad, automarginación, depresión.

- La mayor credibilidad hacia los hombres también da como resultado que, a la hora de elegir representantes o personas con poder de decisión (tribunales, jefes,...), la tendencia sea a escoger hombres.

- Otra consecuencia, y muy grave, es que al valorar un curriculum no cuenta lo mismo, a igualdad de méritos objetivos, si pertenece a un científico o a una científica, por supuesto siempre a favor del primero. Desafortunadamente, debido a su mayor credibilidad, el hombre viene dotado de manera natural de algunos puntos extra «subjetivos» que se suman al total. Sus trabajos serán «mejores» y sus contribuciones en una colaboración serán "más importantes». $\mathrm{Y}$ lo peor es que nadie en el tribunal necesita expresar este tipo de argumentos en voz alta, porque se dan de manera inconsciente. Por ejemplo, es bien sabido que en un trabajo en colaboración entre hombres y mujeres, ellos se llevan el crédito de las ideas relevantes, creatividad y resultados importantes, mientras ellas tienden a ser vistas como meras "ayudantes» a sus órdenes que participan en la parte técnica menos creativa. La regla general en una oposición, salvo en raras circunstancias, es que se dé la plaza a una mujer sólo si sus méritos están muy por encima de los méritos de los candidatos masculinos. Ella debe demostrar su superioridad de manera clarísima.

También la mayoría coincide en que somos diferentes los hombres y las mujeres en nuestra psicología, metodología, formas de presentarnos y perspectiva de la vida. Todo ello nos desfavorece a la hora de triunfar y «hacer carrera» en un mundo laboral dominado por la psicología y maneras de hacer masculinas y donde los valores femeninos no están precisamente en alza. (Se cuentan incluso anécdotas de que para descalificar las diferencias de opinión de sus colegas femeninas algunos hombres 


\section{Mujeres en Ciencia y Tecnologías Físicas en el CSIC}

usan frases del tipo «esas actitudes y posturas son típicas de mujeres»). Nuestra feminidad, que como vemos va en contra de nosotras en lo profesional, suele ser, sin embargo, una ventaja para las personas que trabajan bajo nuestra dirección, debido a nuestra mayor sensibilidad y humanidad y a nuestro ego menos inflado y menos vulnerable. Sin querer pecar de psicólogas amateur, también es interesante señalar que entre las mujeres físicas es bastante común la sensación de que nosotras somos más realistas y equilibradas que nuestros colegas varones y recibimos nuestros éxitos con mayor cordura y naturalidad. En general, los consideramos como un justo premio a nuestro esfuerzo continuado, y en raras ocasiones pensamos que el éxito obtenido es atribuible a que somos especiales y obviamente geniales.

Muchas de nosotras tenemos la impresión de que en el CSIC, como en la Universidad, existen grupos o zonas machistas así como no machistas (grosso modo, ya que todos padecemos en mayor o menor medida el «machismo inconsciente»), siendo más o menos mitad y mitad en número. Así, no es de extrañar que, entre las aportaciones recibidas, se encuentren todo tipo de experiencias, sobre todo en relación a los primeros años de la carrera científica. Las compañeras que cayeron en «territorio machista» desde el principio de sus carreras, cuentan experiencias diferentes de aquellas que cayeron en "territorio no machista». De entre éstas, algunas, sin embargo, notaron que existía un machismo palpable en otros grupos de investigación cercanos al suyo, mientras que otras se toparon con el machismo años después, cuando ya tenían un cierto nivel.

También muchas de nosotras tenemos la impresión de que existe otro factor de suerte, aparte del caer o no caer en una zona no machista. Lo llamamos el «paraguas masculino» y consiste en tener lazos fuertes, normalmente familiares, con algún miembro del mismo departamento o de un departamento afín. Esta circunstancia -particularmente si la mujer está casada con alguien del grupo- parece paliar bastante los efectos indeseables en grupos machistas, parece contribuir a que se respete más a la mujer, a aceptarla y a tratarla como «uno más», en vez de verla como una extraña que se ha colado en el grupo. En las oposiciones, en general, el "paraguas masculino" no da puntos extra a una candidata, es decir, se la seguirá juzgando como a un candidato masculino con los mismos méritos objetivos (o como menos competente que éste, como apuntábamos más arriba), pero sí le facilita el vencer la resistencia natural que cualquier grupo machista opone a la entrada de una mujer, sobre todo en la plantilla fija. Si bien, a la larga, ese paraguas puede volverse en contra de la científica, pudiendo darse el caso de que infravaloren sus méritos precisamente por sus lazos personales con otros colegas. 
Otro factor que nos ayuda a ser más respetadas dentro de un grupo machista es el estatus profesional. Esto sólo lo aprecian con claridad las compañeras que se toparon con grupos machistas desde el comienzo de su carrera científica y que, por tanto, han ido notando los cambios en el trato de sus colegas a medida que ellas han ido mejorando de estatus. En el caso de compañeras que pasaron sus primeros años en grupos no machistas puede darse la impresión contraria, al toparse con el machismo cuando ya gozaban de mayor estatus.

Pasando al capítulo doméstico, la mayoría reconoce que el peso de las obligaciones domésticas (el cuidado del hogar y de los hijos) recae mucho más sobre las mujeres. Es decir, en la mayoría de los casos este peso no está repartido por igual entre el hombre y la mujer. Esta circunstancia puede dar a los hombres más ventajas profesionales, ya que disponen de más tiempo para su trabajo y tienen menos preocupaciones que ocupen su espacio mental. Por ejemplo, será normalmente la mujer quien lleve a los niños al médico, quien les compre la ropa, ordene los armarios y un largo etcétera de obligaciones de las que ellos se evaden sistemáticamente.

Un tema relacionado con el anterior es el de la maternidad y las guarderías. La gran mayoría de las compañeras con hijos se quejan de la insuficiente infraestructura en guarderías, que no facilita la compaginación de ser científica y madre al mismo tiempo. En particular, el CSIC tendría que hacer un esfuerzo en apoyar a los padres y madres con hijos pequeños, haciéndose con más plazas de guardería, menos discriminación por ingresos económicos y más horas de apertura para permitir una flexibilidad de horario que es importante cuando se trabaja en investigación científica. Respecto a la maternidad en sí misma, algunas compañeras han sentido algún tipo de discriminación por parte de algunos colegas por el mero hecho de tener hijos, como si fueran a disminuir su capacidad profesional. Por ejemplo, leemos en una contribución: «Tener un hijo es un argumento que puede ser utilizado para justificar cualquier decisión de mi parte». Por otro lado, afortunadamente las compañeras sin hijos se muestran muy solidarias con las que los tienen. Una de nuestras compañeras, siendo CT, se encontró con una reducción de sueldo de 40000 pesetas en su baja por maternidad en 1999, ya que la Seguridad Social sólo pagaba el tope del nivel y ella ganaba más. Desde que entró en vigor esa legislación, el CSIC se había hecho cargo de la diferencia pero no fue así en 1999, contrariamente a lo que pasaba con las bajas por enfermedad. Finalmente y tras una carta a las altas instancias del CISC, éste reconsideró su postura aunque con un retraso de un año. ¿Es tan raro que las científicas se reproduzcan? ¿Seremos una especie a extinguir sin todavía habernos desarrollado plenamente? 


\section{Mujeres en Ciencia y Tecnologías Físicas en el CSIC}

Por último, ha habido compañeras que nos han comunicado casos de acoso sexual. Si bien este problema no nos consta que sea frecuente, y por tanto no es un hecho generalizable, la gravedad del tema exige un seguimiento serio. Habría que hacer encuestas a las científicas donde este punto se pudiese denunciar de forma anónima para tener una idea exacta de cual es la situación real al respecto.

A continuación, desglosamos resumidamente por etapas en la carrera científica, las dificultades que se detectan en los escritos recibidos sobre experiencias personales de científicas en el Área de Física y Tecnologías Físicas del CSIC. Como se desprende de lo expuesto anteriormente, dichos escritos contienen los siguientes puntos en común:

a) Período predoctoral: Una gran parte asegura no haber notado discriminación por sexo, siendo común a todas ellas el hecho de que en el Grupo de Investigación al que se incorporaban había ya alguna otra mujer y/o su pareja estaba en el mismo centro. Aquellas mujeres que, sin embargo, se adherían en solitario a grupos netamente masculinos sí indican haber sufrido discriminación personal además de escuchar comentarios de género que se consideraban tan normales que se hacían en presencia de la becaria y sin ningún respeto hacia ella.

b) Período Postdoctoral: Todas en sus periodos post-doctorales en países anglosajones pudieron comprobar que la situación de las mujeres científicas en los países mediterráneos era mejor. La discriminación de la mujer en los países anglosajones es manifiesta, abundan los comentarios de género y se caracteriza por la casi total ausencia de mujeres en Física con puesto permanente.

c) Puesto permanente. El problema de las físicas en plantilla está muy bien reflejado en los números que aparecen en la sección 2. Las dificultades en este periodo se resumen a continuación.

- Promoción: Por una parte nos encontramos con que si el numero de mujeres que estudian Físicas no es muy elevado (35\% en 2001, tomando como muestra la UCM), los porcentajes de científicas en plantilla en los centros del Área del CSIC son muy inferiores, como se ve en la sección 2 . Las colegas que nos envían sus experiencias personales hablan de «techo de cristal» para las mujeres, de «una cierta desconfianza o incomodidad por parte de los hombres para elegir mujeres en cargos de responsabilidad», o lo que es lo mismo «que a la hora de elegir a una persona que nos represente o que tenga poder de decisión» dentro del CSIC la tendencia es escoger a un hombre (premios de investigación incluidos). En resumen si ya es sinuosa la carrera científica, para las mujeres hay barreras adicionales y específicas de género. 
- Tribunales y comentarios discriminatorios: Todas coinciden en que en conversaciones generales, tanto el lenguaje como los comentarios particulares tienen sesgo de género, a veces sutil y siempre descalificador. Alguien dice que la diferencia no se nota cuando se escribe un artículo científico sino cuando se habla del éxito científico de colegas mujeres. Tales comentarios discriminatorios en un tribunal, aunque en sí banales, suelen tener un efecto demoledor para la concursante. Una mayor participación de las mujeres en los tribunales, al menos en número igual al porcentaje de mujeres en la escala, reduciría ese tipo de comentarios en una situación tan importante.

- La familia: El tema familiar y de descendencia mencionado en todos los informes es muy recurrente. Los aspectos legales fallan más de lo que uno se atrevería a pronosticar. Si eres becario post-doctoral (en la actualidad, en nuestro campo no osan las mujeres tener hijos en el periodo predoctoral), te encuentras con que la baja maternal consiste en la suspensión de la beca y su correspondiente remuneración. Incluso, ha habido algún caso de anomalías en el sueldo de alguna científica en plantilla por razones de maternidad. Además de los temas laborales hay dos puntos más que se repiten cuando una científica decide tener un hijo: 1) La investigadora parece alcanzar directamente su «techo de cristal». 2) La investigadora se encuentra con el problema de las guarderías. ¿Qué hacer? ¿Cómo compaginar los «horarios de oficina» de la guardería con las largas jornadas de una investigadora? Se vuelve a casa de noche y se utilizan los fines de semana para no dejar de ser madre y científica a la vez, para demostrar que comentarios como «contigo no se podrá contar en unos pocos años» no son verdad. Si todos coincidimos en que la investigación es una profesión de alta dedicación y sacrificio, el CSIC debería facilitar a sus investigadoras guarderías que contemplen horarios hasta las $19 \mathrm{~h}$, permitiéndoles compaginar con mayor facilidad la maternidad con la dedicación a la investigación. Parece que tener un hijo y ser mujer lleva implícito una renuncia al éxito profesional. No se puede negar que tener un hijo repercute temporalmente en la dedicación profesional, tanto del hombre como de la mujer, pero si bien al hombre se considera que lo hace más ambicioso a la mujer se presupone que ya se «realiza» con la maternidad y que el éxito profesional por el que tan duramente ha trabajado no le interesa. 


\section{Mujeres en Ciencia y Tecnologías Físicas en el CSIC}

\section{Comentarios finales y recomendaciones}

La situación de la mujer en el CSIC y particularmente en nuestra área, nos parece un reflejo de la sociedad en que vivimos. Formalmente existe igualdad de derechos, pero la inercia histórica hace que, «de facto», la mujer no tenga la misma relevancia que el hombre, especialmente en los niveles más altos y en los círculos de «toma de decisiones». Tanto las estadísticas que hemos realizado como las aportaciones personales recogidas, nos hablan de la existencia de «un techo de cristal». En nuestra carrera profesional éste aparece desde el acceso a la primera escala permanente (Científico Titular) y se acentúa aún más a partir de ella.

Las estadísticas revelan que el porcentaje de mujeres en nuestra área es significativamente inferior a la media del CSIC, no habiendo mejorado en los últimos diez años. Este hecho podría deberse en parte a la menor participación de la mujer en la Física desde la etapa de licenciatura. Sin embargo, hay indicadores de que el porcentaje de físicas en el CSIC es a su vez inferior al correspondiente porcentaje de estudiantes de licenciatura. El análisis de las causas de ese sesgo inicial merecería ser estudiado con más detalle (¿es la sociedad la que desanima a la mujer por el camino de la investigación en Física?, ¿es el ambiente universitario o de los centros de investigación?...). Los testimonios y opiniones personales nos muestran la «cara humana» de las estadísticas. Además de un escaso estímulo para que las mujeres se inicien en la Física, las que finalmente entran en el CSIC y quieren progresar profesionalmente, encuentran un «techo invisible».

El CSIC debe aceptar su papel motor en el desarrollo científico y cambio social. Para que pueda desarrollar una Ciencia de Excelencia es necesario que favorezca una Ciencia sin género, aprovechando las capacidades de todas las personas y realizando políticas activas para eliminar los sesgos existentes, particularmente en nuestra Área. Por tanto creemos que nuestra Institución debería diseñar acciones positivas para provocar un punto de inflexión en la participación de mujeres en la investigación científica en nuestra Área. He aquí nuestras recomendaciones:

- Realización de un estudio estadístico profundo de la situación de la mujer en CSIC, y en particular en nuestro área, donde, con los datos a los que hemos tenido acceso, parecen existir claras diferencias en las distintas especialidades. Sería necesaria la creación de un grupo de trabajo que, dotado con medios, continuara la labor empezada por los grupos que han presentado los primeros resultados de género en el CSIC y las aportaciones recogidas en este documento. Este estudio debería partir de la realización de una encues- 


\section{Elvira Moya de Guerra}

ta lo más exhaustiva posible tanto en el aspecto profesional (historial profesional, contribuciones científicas, participación en comisiones y órganos de decisión sobre política científica...) como en aspectos personales (número de hijos/hijas, edades, diferencias, existencia de presiones...) e incluyendo todos los niveles (becarias predoctorales, post en estancias fuera de España, post en el CSIC, contratadas posdoctorales, CT, TS, IC, PI...). Este estudio, con datos sistemáticos y con una visión retrospectiva, se vislumbra como una de las mejores maneras de proporcionar las claves sobre el tipo de desequilibrios existentes y sobre los procesos que conducen a ellos en las diferentes áreas de conocimiento $\mathrm{y}$, dentro de éstas, en las diferentes especialidades y líneas de investigación.

- Elaboración sistemática de estadísticas desglosadas por género. Es necesario que el CSIC incluya la variable género de manera sistemática en sus estudios e informes y en las estadísticas de solicitudes/concesiones de becas, contratación, acceso a la escala investigadora, promoción, tribunales, comisiones, etc., incluyendo todas las etapas y niveles administrativos de la carrera científica (desde predoctoral hasta Profesores de Investigación), de manera que se pueda analizar cómo cambian esas proporciones en las distintas líneas del área y si es necesario incidir especialmente en alguna de las especialidades existentes. Esta elaboración sistemática, publicada periódicamente, sería la base fundamental tanto para conocer la situación actual de la Mujer en la Física del CSIC y sus perspectivas de mejora en los próximos años, como para dar indicaciones de si son necesarias acciones positivas encaminadas a integrar la igualdad en las estructuras organizativas, de gestión y de política científica del CSIC.

- Política activa de participación. El CSIC debe asegurar una mayor y apropiada participación y representación de las científicas del área en los diferentes foros de toma de decisiones y de política científica: órganos directivos, comisiones de selección, tribunales, comités de expertos, representaciones a nivel institucional, etc. De todos los datos recogidos podemos afirmar que las mujeres en el área de Física se encuentran claramente subrepresentadas y resulta preocupante el escaso nivel de elección de científicas del área en los puestos de decisión de la política científica del CSIC, uno de cuyos primeros ejemplos son los tribunales y comisiones de selección. Los tribunales son, sin duda, una pieza clave en la política científica de una institución. No es justificable que, siendo pocas las científicas de un determinado rango, no estén ni siquiera re- 


\section{Mujeres en Ciencia y Tecnologías Físicas en el CSIC}

presentadas proporcionalmente en los tribunales. La dimensión de género debería tenerse en cuenta en el diseño de los programas de actuación, como se está implantando ya en la Unión Europea ${ }^{5,6}$.

- Apoyo a la maternidad. El CSIC debe realizar un programa con las actuaciones necesarias para facilitar la compatibilidad de la carrera científica y la familia, de modo que el hecho de tener hijos no sea perjudicial para la promoción de las mujeres. Un aspecto importante es la creación de guarderías y centros escolares con horario flexible, donde para la admisión de los hijos no se penalice a los padres por sus ingresos económicos. Otro aspecto es la flexibilización de los límites de edad o tiempo en las convocatorias de becas, contratos postdoctorales, etc. La organización actual del trabajo científico se ajusta a modelos de trabajo masculinos, con tiempo casi ilimitado dedicado a la investigación y en los que tradicionalmente la dedicación a la familia es mucho menor, lo que claramente perjudica a las mujeres científicas.

- Políticas de visibilidad y motivación. Difusión del papel que ha jugado y juega la mujer en la Física, con objeto de educar a la sociedad y de motivar a las futuras generaciones. La NASA, por ejemplo, está actualmente llevando a cabo un programa en este sentido*. Esta política debe dirigirse a todos los niveles educativos, para eliminar el estereotipo de que la ciencia es «cosa de hombres».

Mencionemos para terminar el artículo de V. Verdú, titulado «El regreso de los valores familiares» (El País, domingo 3/2/2002, Tendencias 11). Dice que «tras la masculinización femenina emerge una feminización de la virilidad», que según el autor consiste en que «antes había que llegar a ser alguien, ahora se trata de llegar a ser Yo». Pues bien: si logramos transmitir a los hombres, en nuestro trabajo codo con codo, el interés en el trabajo bien hecho y en la familia muy por delante del éxito a cualquier precio, la sociedad habrá dado un paso de gigante.

\section{Agradecimientos}

Son muy numerosos los colegas, personal de apoyo y amigos que nos han ayudado en el curso de este trabajo. A todos ellos queremos desde aquí expresar nuestros agradecimientos. Especial mención requieren:

Susana Jiménez (sin cuyo apoyo técnico este trabajo no hubiese sido posible) y, María Egüés, Luisa González, Berta Rubio, María Luisa Se-

\footnotetext{
* http://www.jpl.nasa.goc/webcas/womeniscience.html
} 
nent, Isabel Tanarro y Carmen Torras, que nos enviaron por escrito opiniones y/o experiencias, así como a Carmen Vela que nos facilitó informes de la Comisión Europea.

También queremos agradecer a Concha Domingo, Mar García, Priscila García, Susana Marcos, Carmen Refolio, Rosalía Serna y Carmela Valdemoro, la manifestación de sus opiniones en distintas conversaciones.

Finalmente, una de nosotras (E.M.G.) está muy agradecida a M.A. Durán y C. Moya por sus enseñanzas sobre Sociología.

\section{Apéndice}

Centros e Institutos de Investigación del Área de Ciencia y Tecnologías Físicas

CAB Centro de Astrobiología

CETEF Centro de Tecnologías Físicas «L. Torres Quevedo»

CFMAC Centro de Física «Miguel A. Catalán»

CNA Centro Nacional de Aceleradores

CNM Centro Nacional de Microelectrónica

CSIC-REDIRIS Centro de Comunicaciones

CTI Centro Técnico de Informática

IA Instituto de Acústica

IAA Instituto de Astrofísica de Andalucía

IAG Instituto de Astronomía y Geodesia

IAI Instituto de Automática Industrial

ICE Instituto de Ciencias del Espacio

IEM Instituto de Estructura de la Materia

IFA Instituto de Física Aplicada

IFIC Instituto de Física Corpuscular

IFCA Instituto de Física de Cantabria

IIIA Instituto de Investigación en Inteligencia Artificial

IMAFF Instituto de Matemática y Física Fundamental

IMB-CNM Instituto de Microelectrónica de Barcelona

IMM-CNM Instituto de Microelectrónica de Madrid

IMS-CNM Instituto de Microelectrónica de Sevilla

IO Instituto de Optica 'Daza de Valdés'

IRII Instituto de Robótica e Informática

IMEDEA Instituto Mediterráneo de Etudios Avanzados

OE Observatorio de Física Cósmica del Ebro 


\section{Bibliografía}

1 A. Colosimo, B. Degen,y N. Dewandre, Editores. «Women in Science: Making change happen"; Proc. of the Conference. European Comission, Luxemburgo, 2001.

2 Lise MeITNER, "Looking back». Bulletin of the Atomic Scientists (Nov. 1964).

3 Sharon Bertsch Mc Grayne, «Nobel Prize Women in Science». Joseph Henry Press, Washington D.C., 1998.

4 Mary Harrington Hall, "María Mayer: The Marie Curie of the Atom». Mc Calls (Julio 1964) 91; «The Nobel Genius», San Diego Magazine (Agosto 1964).

5 MARY OsBorn y Col. (Red ETAN), «Promover la excelencia mediante la integración de la igualdad entre géneros" (Informe de "Política científica de la Unión Europea»); Comisión Europea, Bruselas (2001).

6 Christine Wenneras y Agnes Wold, Nature 387 (1997) 341. 